\title{
MUON RECONSTRUCTION AND IDENTIFICATION FOR THE EVENT FILTER OF THE ATLAS EXPERIMENT
}

\author{
A. VENTURA* ${ }^{*}$ on behalf of the ATLAS TDAQ-HLT group ${ }^{\dagger}$
}

The ATLAS Trigger requires high efficiency and selectivity in order to keep the full physics potential of the experiment and to reject uninteresting processes from the $40 \mathrm{MHz}$ event production rate of the LHC. These goals are achieved by a trigger composed of three sequential levels of increasing accuracy that have to reduce the output event rate down to $\sim 100 \mathrm{~Hz}$. This work focuses on muon reconstruction and identification for the third level (Event Filter), for which specific algorithms from the off-line environment have been adapted to work in the trigger framework. Two different strategies for accessing data are described and their reconstruction potential is shown in terms of efficiency, resolution and fake muon rejection power.

\footnotetext{
* Contact author: Andrea.Ventura@le.infn.it - INFN Sezione di Lecce, via per Arnesano, 73100 Lecce, Italy. $\dagger_{\text {A. dos Anjos }}^{a}$, S. Armstrong ${ }_{b}$, J.T.M. Baines ${ }^{c}$, C.P. Bee ${ }^{d}$, M. Bellomo ${ }^{e}$, M. Biglietti ${ }^{f}$, J. A. Bogaerts ${ }^{g}$, M. Bosman $^{h}$, G. Carlino ${ }^{f}$, B. Caron ${ }^{i}$, P. Casado ${ }^{h}$, G. Cataldi ${ }^{j}$, D. Cavalli ${ }^{k}$, G. Comune ${ }^{l}$, P. Conde ${ }^{g}$, F. Conventi ${ }^{f}$, G. Crone $^{m}$, D. Damazio $^{b}$, A. De Santo ${ }^{n}$, M. Diaz Gomez ${ }^{o}$, A. Di Mattia ${ }^{p}$, N. Ellis $^{g}$, D. Emeliyanov ${ }^{c}$, B. $\operatorname{Epp}^{q}$, S. Falciano ${ }^{p}$, H. Garitaonandia ${ }^{h}$, S. George ${ }^{n}$, V. Ghete ${ }^{q}$, S. Goncalo ${ }^{n}$, E. Gorini ${ }^{j}$, S. Grancagnolo ${ }^{j}$, J. Haller ${ }^{g}$, S. Kabana ${ }^{r}$, A. Khomich ${ }^{s}$, G. Kilvington ${ }^{n}$, N. Kirk ${ }^{c}$, N. Konstantinidis ${ }^{m}$, A. $\operatorname{Kootz}^{t}$, A. J. Lankford ${ }^{u}$, A. Lowe ${ }^{n}$, L. Luminari ${ }^{p}$, T. Maeno ${ }^{b}$, J. Masik $^{v}$, C. Meessen $^{d}$, A. G. Mello ${ }^{w}$, R. Moore ${ }^{i}$, P. Morettini ${ }^{x}$, A. Negri ${ }^{e}$, N. Nikitin ${ }^{y}$, A. Nisati ${ }^{p}$, C. Osuna ${ }^{h}$,

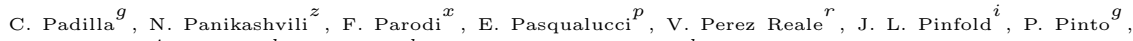
M. Primavera ${ }^{j}$, Z. Qian ${ }^{d}$, S. Resconi ${ }^{k}$, S. $\operatorname{Rosati}^{g}$, C. $\operatorname{Sanchez}^{h}$, C. Santamarina ${ }^{g}$, D. A. Scannicchio ${ }^{e}$ C. Schiavi ${ }^{x}$, E. Segura ${ }^{h}$, J. M. de $\operatorname{Seixas}^{w}$, A. $\operatorname{Sidoti}^{p}$, G. Siragusa ${ }^{j}$, S. Sivoklokov ${ }^{y}$, A. Sobreira ${ }^{g}$, R. Soluk ${ }^{i}$, S. Spagnolo ${ }^{j}$, E. Stefanidis ${ }^{m}$, S. Sushkov ${ }^{h}$, M. Sutton ${ }^{m}$, S. Tapprogge ${ }^{a a}$, S. Tarem ${ }^{z}$, E. Thomas ${ }^{r}$, F. Touchard ${ }^{d}$, G. Usai ${ }^{b b}$, B. Venda Pinto ${ }^{c c}$, A. Ventura ${ }^{j}$, V. Vercesi ${ }^{e}$, T. Wengler ${ }^{g}$, P. Werner ${ }^{g}$, S. J. Wheeler ${ }^{i, u}$, F. J. Wickens ${ }^{c}$, W. Wiedenmann ${ }^{a}$, M. Wielers ${ }^{c}$, G. Zobernig ${ }^{a} \cdot{ }^{a}$ Department of Physics, University of Wisconsin, Madison, Wisconsin, USA, ${ }^{b}$ Brookhaven National Laboratory (BNL), Upton, New York, USA, ${ }^{c}$ Rutherford Appleton Laboratory, Chilton, Didcot, UK, ${ }^{d}$ Centre de Physique des Particules de Marseille, IN2P3-CNRS-Université d'Aix-Marseille 2,

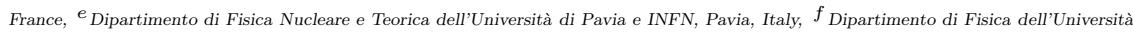
degli Studi di Napoli "Federico II" e INFN, Napoli, Italy, ${ }^{g}$ CERN, Geneva, Switzerland, ${ }^{h}$ Institut de Física d'Altes Energies (IFAE), Universidad Autónoma de Barcelona, Barcelona, Spain, ${ }^{i}$ University of Alberta, Edmonton, Canada, ${ }^{j}$ Dipartimento di Fisica dell'Università degli Studi di Lecce e INFN, Lecce, Italy, ${ }^{k}$ Dipartimento di Fisica dell'Università di Milano e INFN, Milan, Italy, ${ }^{l}$ University of Michigan, Ann Arbor, Michigan, USA, ${ }^{m}$ Department of Physics and Astronomy, University College London, London, UK, ${ }^{n}$ Department of Physics, Royal Holloway, University of London, Egham, UK, ${ }^{o}$ Section de Physique, Université de Genève, Switzerland, ${ }^{p}$ Dipartimento di Fisica dell'Università di Roma 'La Sapienza' e INFN, Rome, Italy, ${ }^{q}$ Institut für Experimentalphysik der Leopald-Franzens Universität, Innsbruck,

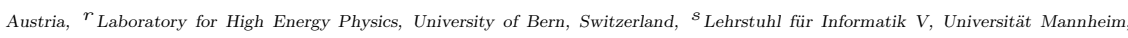
Mannheim, Germany, ${ }^{t}$ Fachbereich Physik, Bergische Universität Wuppertal, Germany, ${ }^{u}$ University of California at Irvine, Irvine, USA, $v^{v}$ Institute of Physics, Academy of Sciences of the Czech Republic, Prague, Czech Republic, ${ }^{w}$ Universidade Federal do Rio de Janeiro, COPPE-EE, Rio de Janeiro, Brazil, ${ }^{x}$ Dipartimento di Fisica dell'Università di Genova e INFN, Genoa, Italy, ${ }^{y}$ Institute of Nuclear Physics, Moscow State University, Moscow, Russia, ${ }^{z}$ Department of Physics, Technion, Haifa, Israel, ${ }^{a a}$ Institut für Physik, Universität Mainz, Mainz, Germany, ${ }^{b b}$ Dipartimento di Fisica dell'Università di Pisa e INFN, Pisa, Italy, ${ }^{c c}$ CFNUL - Universidade de Lisboa, Faculdade de Ciências, Lisbon, Portugal.
} 


\section{Introduction}

The ATLAS experiment (A Toroidal LHC ApparatuS) ${ }^{1}$ is a multi-purpose experiment to run at the LHC (Large Hadron Collider), the new accelerator facility under construction at CERN, the European Laboratory for Particle Physics in Geneva, Switzerland. At its design luminosity $\left(10^{34} \mathrm{~cm}^{-2} \mathrm{~s}^{-1}\right)$, the LHC will provide about 23 inelastic proton-proton collisions for each bunch crossing at a center of mass energy of $14 \mathrm{TeV}$.

The ATLAS detector is composed of concentric shells of specialized sub-detectors arranged in a cylindrical symmetry around the beam axis: an inner tracking detector inside a solenoidal magnetic field of about $2 \mathrm{~T}$, a calorimetric system for energy measurements and a muon spectrometer (extending for $42 \mathrm{~m}$ in length and $22 \mathrm{~m}$ in diameter) in a large air-core toroidal magnetic field.

\section{The ATLAS Trigger DAQ System}

The ATLAS Trigger and Data Acquisition (TDAQ) system has to face the unprecedented rate of $10^{9}$ interactions per second and to reduce this to a final event rate of the order of $100 \div 200 \mathrm{~Hz}$ (as imposed by the limited storage data flow), being able to select rare physics events while rejecting the huge amount of background expected at the LHC. To achieve this goal, the ATLAS TDAQ system is structured in three levels (Figure 1): each level has to refine the hypotheses formed at the previous one.

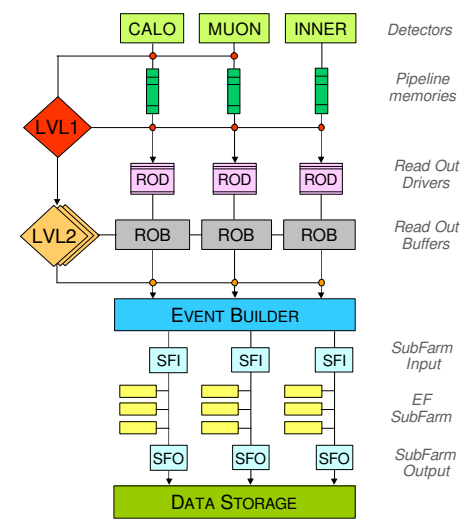

Figure 1. Block diagram of the three-level ATLAS Trigger/DAQ system.

The first trigger level (LVL1) ${ }^{2}$ is implemented with electronic modules directly connected to calorimeters and muon detectors. It has to reduce the 
$40 \mathrm{MHz}$ bunch crossing rate to $75 \mathrm{kHz}$ (upgradable to $100 \mathrm{kHz}$ ) within a $\sim 2.5 \mu$ s fixed latency. At this stage Regions of Interest (RoIs) are defined, i.e. parts of the apparatus where relevant physics signatures are detected.

The amount of data are then transmitted to the High Level Triggers (LVL2 and Event Filter) ${ }^{3}$, that run on commercial computer farms and are fully software-based. The LVL2 accesses to the data from the LVL1 RoIs and processes them with fast algorithms optimized for working in a latency of $10 \mathrm{~ms}$ and for reducing the event rate to $\sim 2 \mathrm{kHz}$. The Event Filter starts from the LVL2 selection to perform a more detailed event reconstruction, including alignment and calibration data. It is expected to perform the event selection in $1 \mathrm{~s}$ with an output of the order of $100 \mathrm{~Hz}$. At the end of the selection process, events are finally saved on mass storage.

\section{Muon Identification}

In order to retain events with muons in the final state that can give evidence for important physics processes, the Event Filter has been designed with two complementary software packages entirely developed in $\mathrm{C}++$ for offline reconstruction: MOORE $^{4}$ (Muon Object-Oriented REconstruction) and MuId ${ }^{5}$ (Muon Identification), that use information from the Muon Spectrometer to provide excellent muon reconstruction and identification over a wide range of transverse momentum ( $p_{T}$ from few $\mathrm{GeV} / \mathrm{c}$ to $3 \mathrm{TeV} / \mathrm{c}$ ). Monitored Drift Tubes (MDT) and Cathode Strip Chambers (CSC) allow the ATLAS tracking system to reach a high-precision measurements, while Resistive Plate Counters (RPC) and Thin Gas Chambers (TGC) are used to provide the LVL1 trigger.

The MOORE package starts searching relevant activities in the Muon Spectrometer volume, subsequently running pattern recognition and track fitting. Owing to the toroidal magnetic field, charged particles are bent in the $r$ - $z$ plane and not in the $\phi$ view: for this reason MOORE firstly looks for straight segments from the $\phi$ trigger hits, and then refines track reconstruction by considering the precision hits in the $r-z$ view.

MuId runs in two steps. At the beginning, it refits the tracks reconstructed by MOORE to obtain their parameters at the production vertex (MuId StandAlone); propagation through the magnetic field, multiple scattering and energy loss in the calorimeters are properly taken into account. In a second step (MuId Combined), tracks in the Muon Spectrometer are matched together with those found in the Inner Detector by the iPatRec package ${ }^{6}$ performing a combined fit, and when matches with $\chi^{2}$ probability greater than $10^{-3}$ are found, these are finally kept as identified muons. 


\subsection{MOORE and MuId in the triggering process}

To use all the required software components in the trigger environment and to avoid dependencies on the off-line, the algorithms for the Muon Event Filter have been isolated in the TrigMoore package ${ }^{7}$ and implemented to run both in a "wrapped" mode (e.g. executing MOORE/MuId as in the off-line on the full muon spectrometer) and in a "seeded" mode (namely performing reconstruction only in given RoIs defined at earlier trigger stages).

\section{Reconstruction performance and background rejection}

Detailed studies on MOORE and MuId have been performed on single muon Monte Carlo samples of different $p_{T}$ 's. Fig. 2 shows the $1 / p_{T}$ resolutions and the efficiencies obtained with the off-line versions of the algorithms. MuId Combined provides the best $p_{T}$ resolution over the full $p_{T}$ range since
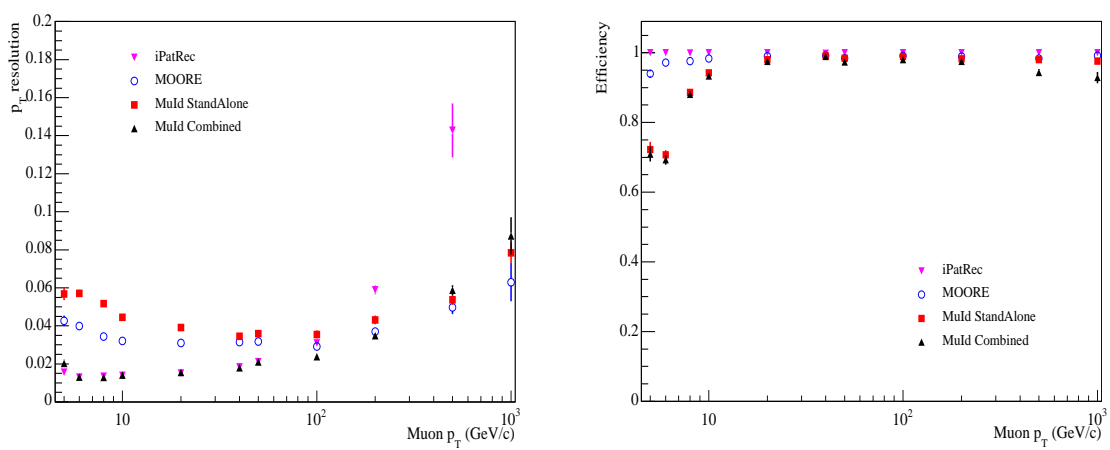

Figure 2. Transverse momentum resolution (left) and reconstruction efficiency (right) for the algorithms MOORE, MuId StandAlone, iPatRec and MuId Combined.

it exploits both Inner Detector and Muon Spectrometer measurements.

The main sources of muon rate in the LVL1 trigger are in-flight decays of charged $K$ and $\pi$. The HLT aims to reject such fake muons while keeping high efficiency on prompt muons (mainly from $b$ and $c$ decays ${ }^{8}$ ). This is achieved through MuId Combined, asking for MuId StandAlone tracks with small impact parameters that match with the Inner Detector tracks. In Fig. 3 (left) the recostruction efficiency is shown for prompt muons and for decay muons in the low $p_{T}$ region: differential muon trigger rates computed at $p_{T}>6 \mathrm{GeV} / \mathrm{c}$ are observed to be $\sim 2.5$ times larger from prompt muons than from $K / \pi$ in-flight decays. Trigger rates for single muon events with $p_{T}>20 \mathrm{GeV} / \mathrm{c}$ have been recently estimated to be $\sim 180 \mathrm{~Hz}$ (fake muon contamination being at a few \% level). Further rate reduction should be accomplished by asking for muon isolation and by combining single muons 

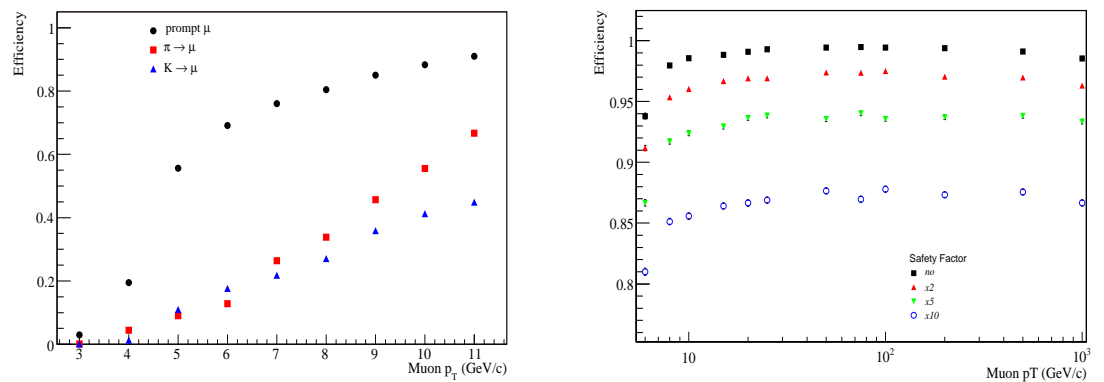

Figure 3. MuId Combined efficiency versus $p_{T}$ for prompt $\mu$ and for $\mu$ from $\mathrm{K} / \pi$ decays (left). MuId StandAlone efficiency for prompt muons at different transverse momenta without and with addition of different amounts of background (right).

with other signatures to select interesting events (for example other leptons to select multi-lepton final state Higgs decay modes).

Another source of noise is the uncorrelated cavern background ${ }^{1}$ expected in the ATLAS experimental area, which has been simulated and extensively studied with TrigMoore, also in terms of execution times ${ }^{7}$; the plot on the right of Fig. 3 shows the MuId StandAlone efficiency on simulated muon samples of different $p_{T}$, both without and with background (the "nominal" background intensity has been increased by factors $\times 2, \times 5, \times 10)$.

\section{Conclusions}

In this work the implementation and the performance of the ATLAS muon off-line reconstruction packages MOORE and MuId in the trigger environment are discussed. The results described here demonstrate that they can be successfully used as Event Filter algorithms.

\section{References}

1. ATLAS collab., ATLAS Detector and Physics Performance Technical Design Report, CERN-LHCC 99-14 and 99-15, 1999.

2. ATLAS collab., ATLAS Level-1 Trigger: Technical Design Report, CERNLHCC-98-014, ATLAS-TDR-12, June 1998.

3. ATLAS collab., ATLAS High-Level Triggers, DAQ and DCS Technical Proposal, CERN-LHCC-2000-17, March 2000.

4. J. Shank et al., Track Reconstruction in the ATLAS Muon Spectrometer with MOORE, ATL-COM-MUON-2003-012, ATL-COM-SOFT-2003-007.

5. Th. Lagouri et al., A Muon Identification and Combined Reconstruction Procedure for the ATLAS Detector, IEEE Trans.Nucl.Sci. Vol.51 \#6 (2004), 3030.

6. R. Clifft and A. Poppleton, IPATREC: Inner Detector Pattern-Recognition and Track Fitting, ATL-SOFT-94-009.

7. D. Adams et al., Moore as Event Filter in the ATLAS High Level Trigger, ATL-DAQ-2003-012, 2003.

8. ATLAS Muon collab., ATLAS Muon Spectrometer Technical Design Report, CERN/LHCC 97-22, May 1997. 